\title{
Antibiotic Susceptibility Pattern of Salmonella enterica serovars Typhi and Paratyphi A Isolated from Patients Suspected of Enteric Fever
}

\author{
Dhirendra Niroula ${ }^{1}$, Jyotsna Shrestha ${ }^{2}$, Supriya Sharma ${ }^{1}$, Anjana Singh ${ }^{1}$, \\ ${ }^{1}$ Central Department of Microbiology, Tribhuvan University, Kirtipur, Kathmandu, Nepal \\ ${ }^{2}$ Bir Hospital, Kathmandu, Nepal
}

\begin{abstract}
Corresponding author: Supriya Sharma, Central Department of Microbiology, Tribhuvan University, Kirtipur, Kathmandu, Email: suprisharma@hotmail.com
\end{abstract}

\begin{abstract}
Objectives: The study aimed to assess the antibiotic susceptibility profile of Salmonella spp isolated from patients suspected of enteric fever.

Methods: This cross-sectional prospective study was carried out from April to June, 2014among 484 patients clinically suspected of enteric fever visiting Bir Hospital, Kathmandu, Nepal. Blood sample collected from each patient was processed for culture in bile broth. Identification of Salmonella spp was done by conventional microbiological techniques including colony characteristics, Gram's staining and biochemical tests. Antibiotic susceptibility testing of identified isolates was done by Kirby-Bauer disk diffusion method following the 2014 CLSI guideline.
\end{abstract}

Results: Out of 484 blood samples, $36(7.43 \%)$ cases showed the growth of Salmonella spp. of which 27 (75\%) were Salmonella enterica serovar Typhi (ST) and $9(25 \%)$ were Salmonella enterica Paratyphi A (SPA). Among the Salmonella isolates, 5.55\% were multidrug resistant and $41.66 \%$ were fluoroquinolone resistant. More than $80 \%$ of isolates were sensitive to chloramphenicol, amoxicillin, and cotrimoxazole whereas $58 \%, 50 \%$ and $6 \%$ of isolates were sensitive to fluoroquinolone antibiotics i.e. ciprofloxacin, ofloxacin and nalidixic acid respectively. All the isolates were susceptible to ceftazidime. All SPA and $89 \%$ of ST were sensitive to azithromycin.

Conclusion: Higher percentage of susceptible isolates to chloramphenicol, cotrimoxazole, and amoxicillin suggests the reconsideration of these antibiotics for the treatment of enteric fever. Azithromycin can be considered as drug of choice for the treatment of enteric fever.

Keywords: Enteric fever, Salmonella isolates, MDR, antibiotic susceptibility

\section{INTRODUCTION}

Enteric fever, a febrile disease caused by the Salmonella enterica serovars Typhi and Paratyphi A, B, and C, causes 21 million new infections and claims 161,000 lives each year worldwide (WHO 2019). It is a systemic disease, endemic in developing countries like Nepal (Crump and Mintz 2010). Definitive diagnosis of enteric fever is done by the isolation of organisms from the blood and bone marrow (Gasem et al. 1995). Serological tests (Widal test) can also be done, however, they are not reliable due to false-positive results. DNA probes and

Date of Submission: August 22, 2020

Published Online: December, 2020
PCR can be used to detect organism from the blood, however, their use in the developing countries is not feasible due to high cost (Parry et al. 2002).

Initially, ampicillin, chloramphenicol, and trimethoprim-sulphamethoxazole (cotrimoxazole) had been used as a first-line drug for the treatment of enteric fever. Salmonella enterica serovars Typhi and Paratyphi resistant to these three first-line antibiotics are called as multidrug-resistant (MDR) strains (Crump et al. 2015). With the increase in MDR strains, fluoroquinolones

Date of Acceptance: November 2, 2020

DOI: https:/ / doi.org/10.3126/tujm.v7i0.33791 
(ofloxacin, nalidixic acid, ciprofloxacin etc.) became the drug of choice for the treatment of enteric fever. However, the dramatic increase in fluoroquinoloneresistant strains has been observed after 2000 (Mirza and Khan 2008). Therefore, third-generation cephalosporins (cefixime, cefalexin, ceftazidime etc.), and azithromycin have been used recently for the treatment of MDR, and fluoroquinolone-resistant Salmonella strains (Effa et al. 2011) though sporadic cases of resistance have been observed for ceftriaxone and azithromycin (Kobayashi et al. 2014, Parry et al. 2015).

In Kathmandu, the burden of the enteric fever is high and is the leading cause of febrile illness (Karkey et al. 2008). Lack of proper diagnosis of disease, on one hand, and continuous development of antimicrobial resistance, on the other hand, are the issues of major concern in the countries of low economic settings like Nepal (Parry et al. 2011). Therefore, this study aims to assess the antibiotic resistance among Salmonella spp isolated from febrile cases in a tertiary care hospital of Kathmandu, Nepal.

\section{MATERIALS AND METHODS}

This cross-sectional prospective study was carried out from April to June, 2014 among 484 clinically suspected enteric fever patients visiting Bir Hospital, a tertiary hospital of Nepal. From each patient, 5-10 ml of blood sample was collected aseptically by vein puncture and inoculated directly into a bottle containing bile broth. Each sample was incubated at $37^{\circ} \mathrm{C}$ for 48 hours and subcultured into Blood Agar (BA) and Mac-Conkey Agar (MA). Each sample was cultured till 7 days to consider as negative. Identification of Salmonella spp was done based on colony characteristics, Gram's staining, catalase, oxidase and other biochemical tests (Cheesbrough 2009). The antibiotic susceptibility pattern of isolates was done in Muller Hinton Agar by Kirby-Bauer disk diffusion method following CLSI guidelines (CLSI 2014). The antimicrobial susceptibility of 10 antimicrobial agents- amoxicillin ( $\mathrm{amx})(30 \mu \mathrm{g})$,

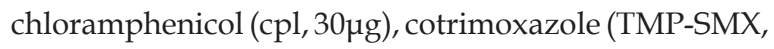

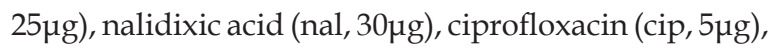

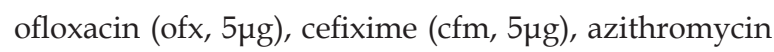

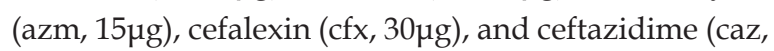
30 $\mathrm{g}$ ) (Hi-Media Laboratory Ltd, Mumbai, India) were performed. The results were interpreted as sensitive, intermediate, or resistant based on the size of zone of inhibition and comparing it with the standard chart provided by the manufacturing company. (CLSI 2014).

\section{RESULTS}

Out of 484 blood samples, 36 (7.43\%) were positive for Salmonella spp of which $27(75 \%)$ were Salmonella enterica serovar Typhi (ST) and 9 (25\%) were Salmonella enterica serovarParatyphi A (SPA).

Most of the isolates were from patients in the age group 21-30 years followed by the age group 11-20 years. Similarly, male patients $(8.27 \%)$ were found to be more susceptible to Salmonella infection than female patients $(6.18 \%)$ but was not statistically significant (Table 1$)$.

Table 1: Age and gender wise distribution of total and positive cases for Salmonella spp

\begin{tabular}{|c|c|c|c|c|c|c|}
\hline \multirow{3}{*}{$\begin{array}{c}\text { Age group in } \\
\text { years }\end{array}$} & \multirow{2}{*}{\multicolumn{2}{|c|}{ Number of cases tested }} & \multicolumn{4}{|c|}{ Number of positive cases } \\
\hline & & & \multicolumn{2}{|c|}{ Salmonella spp } & \multirow{2}{*}{ Salmonella Typhi } & \multirow{2}{*}{$\begin{array}{l}\text { Salmonella } \\
\text { Paratyphi A }\end{array}$} \\
\hline & Male & Female & Male & Female & & \\
\hline$<10$ & 1 & 3 & 0 & 0 & 0 & 0 \\
\hline $11-20$ & 51 & 39 & 7 & 4 & 8 & 3 \\
\hline $21-30$ & 79 & 41 & 15 & 6 & 16 & 5 \\
\hline $31-40$ & 49 & 27 & 0 & 1 & 1 & 0 \\
\hline $41-50$ & 33 & 20 & 1 & 0 & 0 & 1 \\
\hline $51-60$ & 27 & 32 & 0 & 0 & 0 & 0 \\
\hline $61-70$ & 29 & 19 & 1 & 1 & 2 & 0 \\
\hline$>70$ & 21 & 13 & 0 & 0 & 0 & 0 \\
\hline Total & 290 & 194 & 24 & 12 & 27 & 9 \\
\hline
\end{tabular}

More than $80 \%$ of isolates were sensitive to chloramphenicol, amoxicillin, and cotrimoxazole whereas $58 \%, 50 \%$ and $6 \%$ of isolates were sensitive to fluoroquinolone antibiotics i.e ciprofloxacin, ofloxacin and nalidixic acid respectively. All the isolates were sensitive to third-generation cephalosporin, ceftazidime. To azithromycin, $89 \%$ of ST were sensitive and $11 \%$ were intermediately sensitive whereas $100 \%$ of SPA were sensitive (Table 2). 
Table 2: Antibiotic susceptibility pattern of Salmonella isolates

\begin{tabular}{lcccccc}
\hline \multirow{2}{*}{ Antibiotics } & \multicolumn{3}{c}{ Salmonella Typhi (n=27) } & \multicolumn{3}{c}{ Salmonella Paratyphi A (n=9) } \\
\cline { 2 - 7 } & $\begin{array}{c}\text { Sensitive } \mathbf{n} \\
(\%)\end{array}$ & $\begin{array}{c}\text { Intermediate } \\
\mathbf{n}(\%)\end{array}$ & $\begin{array}{c}\text { Resistant } \\
\mathbf{n}(\%)\end{array}$ & $\begin{array}{c}\text { Sensitive } \mathbf{n} \\
(\%)\end{array}$ & $\begin{array}{c}\text { Intermediate } \\
\text { R (\%) }\end{array}$ & $\begin{array}{c}\text { Resistant } \\
(\%)\end{array}$ \\
\hline Amoxycillin & $22(81)$ & - & $5(19)$ & $7(78)$ & - & $2(22)$ \\
Cotrimoxazole & $26(96)$ & - & $1(4)$ & $8(89)$ & - & $1(11)$ \\
Chloramphenicol & $26(96)$ & - & $1(4)$ & $8(89)$ & - & $1(11)$ \\
Ciprofloxacin & $12(44.5)$ & $3(11)$ & $12(44.5)$ & $6(67)$ & - & $3(33)$ \\
Ofloxacin & $11(41)$ & $2(7)$ & $14(52)$ & $5(56)$ & - & $4(44)$ \\
Nalidixic acid & $2(7)$ & - & $25(93)$ & - & - & $9(100)$ \\
Cefixime & $25(93)$ & - & $2(7)$ & $9(100)$ & - & - \\
Cefalexin & $24(89)$ & $3(11)$ & - & $6(67)$ & - & $3(33)$ \\
Ceftazidime & $27(100)$ & - & - & $9(100)$ & - & - \\
Azithromycin & $24(89)$ & $3(11)$ & - & $9(100)$ & - & - \\
\hline
\end{tabular}

Among the Salmonella isolates, 5.55\% were multidrug resistant $\quad$ and $41.66 \%$ were fluoroquinolone resistant (Table 2).

Table 3: Multidrug resistant (MDR) and Fluroquinolone resistant (FQR) Salmonella isolates

\begin{tabular}{lccc}
\hline \multicolumn{1}{c}{ Salmonella serovar } & Total isolates & MDR $\mathbf{n ~ ( \% )}$ & FQR $\mathbf{n}(\%)$ \\
\hline Salmonella Typhi $(\mathrm{n}=27)$ & 27 & $1(3.71 \%)$ & $12(44.44 \%)$ \\
Salmonella Paratyphi A $(\mathrm{n}=9)$ & 9 & $1(11.11 \%)$ & $3(33.33 \%)$ \\
\hline Total & $\mathbf{3 6}$ & $\mathbf{2 ( 5 . 5 5 \% )}$ & $\mathbf{1 5 ( 4 1 . 6 6 \% )}$ \\
\hline
\end{tabular}

\section{DISCUSSION}

Higher prevalence of Salmonella Typhi and Salmonella Paratyphi A in our study was similar to that reported by Petersiel et al. (Petersiel, Shresta et al. 2018).. In contrast, Pokhrel et al. found that the prevalence of ST (47\%) infection was lower than SPA (53\%) (Pokharel et al. 2006). A similar study reported that the paratyphoid fever was associated with flood and contaminated street vendor's food, whereas, typhoid fever was associated with household contamination (Woods et al. 2006). So, we expect most of the patients in our study were associated with household contamination. The proportion of enteric fever positive male patients (66.67\%) was higher than female patients (33.33\%) . A similar study had the proportion of enteric fever positive male patients higher than female patients (Amatya et al. 2007). Males have greater chances of acquiring enteric fever than females, probably due to the indiscriminate eating habits of the male in the roadside locations. The age group 21-30 years had the highest culture positivity $(58.33 \%)$ similar to previous studies in Nepal (Adhikari et al. 2012).

Amoxicillin,cotrimoxazole, and chloramphenicol had been used previously as the first-line drug against the Salmonella infection. However, resistance to these first-line drugs has been reported(Ochiai et al. 2008). We found only $5.55 \%$ of the MDR Salmonella isolates (ST-3.71\%, SPA-11.11\%). Chloramphenicol was used previously (since the 1940s) as a gold standard for the treatment of enteric fever, however, due to the emergence of resistance, it is no longer a drug of choice for the treatment of enteric fever (Mandal et al. 2004). Our study shows that both the serovars of Salmonella were highly susceptible to chloramphenicol (ST-96\%, SPA-89\%) which is in agreement to another similar study conducted in Nepal (Amatya et al. 2007). The sensitivity of ST and SPA toward cotrimoxazole was found to be $96 \%$ and $89 \%$ respectively; similar findings were obtained by two different studies (Murdoch et al. 2004, Amatya et al. 2007). Both the MDR isolates were resistant to cotrimoxazole which is in agreement with the finding of Amatya et al. (100\% resistant) (Amatya et al. 2007). Antibiotic susceptibility pattern of ST and SPA for amoxicillin were $81 \%$ and $78 \%$ respectively, this pattern corresponds with the finding by Amatya et al. (ST- 75\% and SPA- 49\%) (Amatya et al. 2007). Hence, increased susceptibility of ST and SPA towards amoxicillin, cotrimoxazole, and chloramphenicol over the period in Nepal (Karki et al. 2013) mandates the reconsideration of these antibiotics for the treatment of enteric fever.

The resistance to chloramphenicol and amoxicillin during the 1990s led to the extensive use of fluoroquinolones, such as ciprofloxacin, ofloxacin, and nalidixic acid, effective against ST and SPA (Parry et al. 2002). Our study showed that $44.44 \%$ of ST and 
$33.33 \%$ of SPA were resistant to fluoroquinolone antibiotics. Various studies in a different part of the world showed the higher effectiveness of the abovementioned fluoroquinolone antibiotics against ST and SPA (Gales et al. 2002, Wain et al. 2003, Maskey et al. 2008), contradicting our study. However, in agreement with our findings, Pokharel et al. found the decreased susceptibility of ST and SPA against fluoroquinolone(Pokharel et al. 2006). Similarly, Karki et al. in a review article reported the decreased susceptibility of ST and SPA against a range of fluoroquinolone antibiotics (Karki et al. 2013). The susceptibility towards ciprofloxacin (ST- 44.5\%, SPA$67 \%$ ) in our study corresponds to the study by Pokharel et al. (ST- 57\%, SPA- 0\%), while incongruent to a study by Amatya et al. (ST-93.59\%, SPA-79.54\%) (Pokharel et al. 2006, Amatya et al. 2007). The development of resistance towards ciprofloxacin in Nepal is due to the easy availability of ciprofloxacin in the drug store, selfprescription by the patients, and incomplete course of treatment (Pokharel et al. 2006).

Nalidixic acid resistance is considered as the phenotypic marker for the reduced susceptibility to fluoroquinolone. Our study shows that the resistance against nalidixic acid was the highest (ST-93\%, SPA$100 \%$ ) among all the tested antibiotics. In agreement with our study, a recent study was done by Adhikari et al. in the year 2011, found the increased resistance of nalidixic acid antibiotic (ST-82.9\%, SPA-91.33\%) (Adhikari et al. 2012). Similarly, Maskey et al. in 2008 (ST-49\%, SPA-86\%), and Neopane et al. in 2007 (ST$73.3 \%$, SPA-94.9\%) reported the growing resistance of ST and SPA to nalidixic acid antibiotic (Neopane et al. 2007, Maskey et al. 2008). Nalidixic acid had been used as anindicator of decreased ciprofloxacin susceptibility. Our study also shows a similar trend, however, it is suggested that the determination of minimum inhibitory concentration (MIC) as a reliable indicator (Crump et al. 2003).

The third-generation cephalosporins (cefixime, cefalexin, and ceftazidime) were effective against ST and SPA, as the susceptibility of both Salmonella enterica serovars were quite higher. Susceptibility of ST was $89 \%$ and the SPA was $100 \%$ to cefixime.Astudy reported the susceptibility of ST and SPA to cefixime was found to be $75 \%$ and $100 \%$ respectively (Amatya et al. 2007). Cefixime, an oral antibiotic, is widely used for the treatment of enteric fever as a first-line drug (Pandit et al. 2007). As we found two ST isolates resistant against cefixime, which is a worrisome finding, similar to anotherstudy(Qamar et al. 2014). Therefore, further research should be done before blindly prescribing third-generation cephalosporins for the treatment of MDR and FQR Salmonella isolates. Ceftazidime was most effective as both ST and SPA were found to be $100 \%$ susceptible to it. In a similar study done in ST, Hasan et al. reported that ceftriaxone and ceftazidime were the most effective antibiotics ( $100 \%$ susceptibility) (Hasan et al. 2011). However, mode of administration (intravenous and intramuscular) make ceftazidime a less famous antibiotic, with regards to difficulty in administering to the outpatients.

Furthermore, both ST (89\% susceptible, 11\% intermediate susceptible) and SPA (100\% susceptible) are found to be highly susceptible to azithromycin antibiotic. In agreement with our study, Kumar et al. found the susceptibility of ST and SPA to azithromycin to be $93.6 \%$ and $100 \%$ respectively (Kumar et al. 2008). Similarly, another study shows that the antibiotic susceptibility of STwas found to be $81.25 \%$ to azithromycin(Hasan et al. 2011). Another study also highlighted that azithromycin is better in terms of fever clearance, and relapse rate for the treatment of enteric fever caused by MDR and FQR Salmonella isolates (Shah 2009). Hence, our study revealed that azithromycin can be an alternative solution for the MDR and FQR Salmonella isolates.

\section{CONCLUSION}

Higher percentage of susceptible isolates to chloramphenicol, cotrimoxazole, and amoxicillin suggests the reconsideration of these antibiotics for the treatment of enteric fever. Azithromycin can be considered as drug of choice for the treatment of enteric fever.

\section{ACKNOWLEDGEMENTS}

We would like to express sincere gratitude to all the concerned persons of the Central Department of Microbiology, Tribhuvan University, Kirtipur, Kathmandu, Nepal and Bir hospital, Kathmandu, Nepal for their help.

\section{REFERENCES}

Adhikari D, Acharya D, Shrestha P and Amatya R (2012) Ciprofloxacin susceptibility of Salmonella enteric serovar Typhi and Paratyphi A from blood samples of suspected enteric fever patients. 
Int J Infect Microbiol 1(1): 9-13.

Amatya NM, Shrestha B and Lekhak B (2007) Etiological agents of bacteraemia and antibiotic susceptibility pattern in Kathmandu Model Hospital. J Nepal Med Assoc 46(167): 112-118.

Buckle GC, Walker CLF and Black RE (2012) Typhoid fever and paratyphoid fever: systematic review to estimate global morbidity and mortality for 2010 . J Glob Health 2(1):-9.

Cheesbrough M. (2009) District Laboratory practice in tropical countries Part 1. UK, Cambridge University Press.

CLSI (2014) Performance Standards for Antimicrobial Susceptibility Testing; Twenty-Fourth Informational Supplement. CLSI document M100-S24, Clinical and Laboratory Standards Institute.

Crump JA, Barrett TJ, Nelson JT and Angulo FJ(2003) Reevaluating fluoroquinolone breakpoints for Salmonellaenterica serotype Typhi and for nonTyphi salmonellae. Clin Infect Dis 37(1): 75-81.

Crump JA, Gordon MA and Parry CM(2015)Salmonella Infections. Clin Microbiol Rev 28(4): 901-937. doi:10.1128/CMR.00002-15.

Crump JA and Mintz ED(2010) Global trends in typhoid and paratyphoid fever. Clin Infect Dis 50(2): 241-246.

Effa EE, Lassi ZS, Critchley JA, Garner P, Sinclair D, Olliaro PL and Bhutta ZA(2011) Fluoroquinolones for treating typhoid and paratyphoid fever (enteric fever). Cochrane Database Syst Rev 10:1-3.

Gales AC, Sader HS, Mendes RE and Jones RN(2002) Salmonella spp. isolates causing bloodstream infections in Latin America: report of antimicrobial activity from the SENTRY Antimicrobial Surveillance Program (1997-2000). Diagn Microbiol Infect Dis 44(3): 313-318.

Gasem MH, Dolmans WM, Isbandrio BB, Wahyon H, Keuter M and Djokomoeljanto R(1995) Culture of Salmonella Typhi and Salmonella Paratyphi from blood and bone marrow in suspected typhoid fever. Trop Geogr Med 47(4): 164-167.

Hasan B, Nahar SG, Akter L and Saleh AA(2011) Antimicrobial sensitivity pattern of Salmonella
Typhi isolated from blood culture in a referral hospital. Bangladesh J Med Microbiol 5(1): 16-20.

Karkey A, Aryjal A, Basnyat B and Baker S (2008) Kathmandu, Nepal: still an enteric fever capital of the world. J Infect Dev Ctries 2(06):461-465.

Karki S, Shakya P, Cheng AC, Dumre SP and Leder K (2013) Trends of etiology and drug resistance in enteric fever in the last two decades in Nepal: a systematic review and meta-analysis. Clin Infect Dis 57(10): e167-e176.

Kobayashi T, Hayakawa K, Mawatari M, Mezaki K, Takeshita N, Kutsuna S, Fujiya Y, Kanagawa S, Ohmagari N and Kato Y (2014) Case report: failure under azithromycin treatment in a case of bacteremia due to Salmonellaenterica Paratyphi A. BMC Infect Dis 14(1): 404.

Kumar S, Rizvi M and Berry N(2008) Rising prevalence of enteric fever due to multidrug-resistant Salmonella: an epidemiological study. J Med Microbiol 57(10):1247-1250.

Mandal S, Mandal MD and Pal NK (2004) Reduced minimum inhibitory concentration of chloramphenicol for Salmonella enterica serovar Typhi. Medknow Publications on behalf of Indian Journal of Medical Sciences Trust.

Maskey AP, Basnyat B, Thwaites GE, Campbell JI, Farrar JJ and Zimmerman MD (2008) Emerging trends in enteric fever in Nepal: 9124 cases confirmed by blood culture 1993-2003. Trans $R$ Soc Trop Med Hyg 102(1): 91-95.

Mirza SH and Khan MA (2008) Low-level quinoloneresistance in multi-drug resistant typhoid. J Coll Physicians Surg Pak 18(1): 13-16.

Murdoch DR, Woods CW, Zimmerman MD, Dull PM, Belbase RH, Keenan AJ, Scott RM, Basnyat B, Archibald LK and Reller LB (2004) The etiology of febrile illness in adults presenting to Patan hospital in Kathmandu, Nepal. Am J Trop Med Hyg 70(6): 670-675.

Neopane A, Singh SB, Bhatta R, Dhital B and Karki DB(2007) Changing spectrum of antibiotic sensitivity in enteric fever. Kathmandu Univ Med J 6(21): 12-15.

Ochiai RL, Acosta CJ, Danovaro-Holliday M, Baiqing D, Bhattacharya SK, Agtini MD, Bhutta ZA, Canh 
DG, Ali M and Shin S(2008) A study of typhoid fever in five Asian countries: disease burden and implications for controls. Bull World Health Organ 86: 260-268.

Pandit A, Arjyal A, Day JN, Paudyal B, Dangol S, Zimmerman MD, Yadav B, Stepniewska K, Campbell JI and Dolecek C(2007) An open randomized comparison of gatifloxacin versus cefixime for the treatment of uncomplicated enteric fever. PLoS One 2(6).

Parry CM, Hien TT, Dougan G, White NJ.and Farrar JJ(2002) Typhoid fever. N Engl J Med 347(22): 1770-1782.

Parry CM, Thieu NTV, Dolecek C, Karkey A, Gupta R, Turner P, Dance D, Maude RR, Ha V and Tran CN(2015) Clinically and microbiologically derived azithromycin susceptibility breakpoints for Salmonellaenterica serovars Typhi and Paratyphi A. Antimicrob. Agents Chemother 59(5): 2756-2764.

Parry CM, Wijedoru L, Arjyal A and Baker S(2011) The utility of diagnostic tests for enteric fever in endemic locations. Expert Rev Anti Infect Ther 9(6): 711-725.

Petersiel N, Shresta S, Tamrakar R, Koju R, Madhup S and Shresta $S$ (2018) The epidemiology of typhoid fever in the Dhulikhel area, Nepal: A prospective cohort study. PLoS One 13(9): e0204479.

Pokharel BM, Koirala J, Dahal RK, Mishra SK, Khadga PK and Tuladhar N.R. (2006). Multidrug-resistant and extended-spectrum beta-lactamase (ESBL)producing Salmonella enterica (serotypes Typhi and Paratyphi A) from blood isolates in Nepal: surveillance of resistance and a search for newer alternatives. Int J Infect Dis 10(6): 434-438.

Qamar FN, Azmatullah A, Kazi AM, Khan E and Zaidi $\operatorname{AKM}(2014)$ A three-year review of antimicrobial resistance of Salmonellaenterica serovars Typhi and Paratyphi A in Pakistan. J Infect Dev Ctries 8(8):981-986.

Shah D (2009) Role of azithromycin in enteric fever. Indian Pediatr 46: 51-52.

Thapa JB(1991) Drug sensitivity of enteric fever organisms. J. Med 13: 327-330.

Threlfall EJ and Ward LR(1999) Ciprofloxacin-resistant Salmonella Typhi and treatment failure. Lancet 353(9164): 1590-1591.

Wain J, Nga LTD, Kidgell C, James K, Fortune S, Diep TS, Ali T, Gaora PÓ, Parry C and Parkhill J(2003) Molecular analysis of incHI1 antimicrobial resistance plasmids from Salmonella serovar Typhi strains associated with typhoid fever. Antimicrob Agents Chemother 47(9): 273

WHO (2019) Typhoid:Immunization, Vaccines and Biologicals. Retrieved from https://www.who. int/immunization/diseases/typhoid/en/ on $2020 / 8 / 25$

Woods CW, Murdoch DR, Zimmerman MD, Glover WA, Basnyat B, Wolf L, Belbase RH and Reller LB(2006) Emergence of Salmonella enterica serotype Paratyphi A as a major cause of enteric fever in Kathmandu, Nepal. Trans R Soc Trop Med Hyg 100(11): 1063-1067. 